\title{
Pulse Examination by an Ultrasonic Method
}

\author{
S. T. YAO,* M.D. ; J. T. HOBBS,* M.D., F.R.C.S. ; W. T. IRVINE,* M.D., CH.M., B.SC., F.R.C.S.
}

Brit. med.F., 1968, 4, 555-557

Summary : One hundred patients with occlusive arterial $\$$ disease affecting the lower extremities and 25 normal adults were examined by ultrasonic flow velocity detector. This new and harmless method is simple and has proved to be a very useful aid in both the diagnosis and the management of arterial disease.

\section{Introduction}

The most important clinical diagnostic test in the management of peripheral arterial disease is palpation of the distal limb pulses. The recent development of ultrasound techniques has made possible the detection of pulses which are obscured by obesity or by ankle oedema. The blood flow velocity detector obtained both audible and written signals which enable the character of arterial sounds and the flow velocity patterns to be studied.

\section{Theoretical Considerations}

By application of the Doppler effect an ultrasound beam of 10 megacycles per second is coupled to the skin with a gel and then passes through an underlying blood vessel. By detecting and amplifying the frequency shift that is produced by ultrasound passing through moving blood the velocity of flow can be detected. The difference between the transmitted and received frequency is converted to audible signals; a high velocity of blood movement causes a higher-pitched sound than low velocity movement. A time-constant filtering system enables a wave-form tracing of the flow profile to be recorded. The configuration of the wave-form is related to the velocity of the blood flow. The pulsatile patterns of arterial flow velocity can be readily distinguished from the more continuous flow signal from veins. Certain arteries display distinctive flow velocity patterns.

\section{Material and Methods}

During a period of six months 100 patients with occlusive peripheral arterial disease, confirmed by arteriography, and 25 normal adults were included in this study. Fifty patients had aortoiliac occlusion and 50 had femoral and popliteal artery occlusion. In 26 patients undergoing direct arterial reconstruction studies were made before and after surgery.

The transcutaneous blood velocity detector used was the Doppler instrument, model 802, developed by Parks Laboratory. ${ }^{1}$ With the flow probe placed at an angle of 45 degrees to the artery audible and written signals were obtained. Since the instrument does not show the direction of flow, both forward and reversed flow are expressed as a positive deflection in the wave-form tracing.

By using strain-gauge plethysmography, pulse registration at the foot was obtained for comparison with the ultrasound tracings both in normal and in abnormal extremities.

\footnotetext{
* Blood Flow Laboratory, Surgical Unit, St. Mary's Hospital, London W.2.

${ }^{1}$ Parks Laboratories Ltd., Beaverton, Oregon, U.S.A.
}

An ultrasonogram was made in all patients and normal adults by placing the probe over the common femoral, popliteal, posterior tibial, and dorsalis pedis arteries of both legs.

\section{Results}

When the probe was used in the manner of a stethoscope the normal arterial signals could be recognized by the presence of first and second sounds. When this signal was converted to a wave-form a flow velocity pattern with two components, corresponding to systole and diastole, was produced, and this direct record was made by a standard E.C.G. machine. The first signal coincided with systole and the second with the lower frequency produced by reversed flow during diastole, and was then followed by a variable signal which represented the return of forward flow (Fig. 1). When the flow velocity patterns were compared with the plethysmograph tracing distinct second and third flow signals were obtained in legs where a dicrotic notch was recorded by the strain-gauge plethysmograph. When the second and third velocity signals were absent the dicrotic notch was not seen (Fig. 2). When flow signals were detected over an artery distal to the site of an occlusion the first sound was of low pitch and the second sound was usually absent, demonstrating absence of reversed

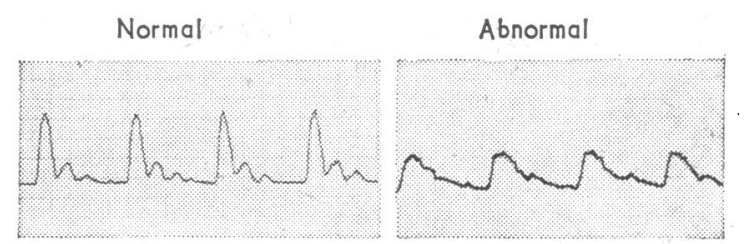

FIG. 1.-Ultrasonic flow signals recorded over the posterior tibial artery. In the normal artery the major deflection indicates forward flow velocity during systole, the second smaller deflection is caused by the lower frequency of reversed flow during diastole, and the small third signal represents the return of for and the small third signal represents the return of forward flow. In an abnorma flow pattern recorded from a patient with a superficial femoral artery occlusion the delayed crest time, lower systolic velocity, and absence of reversed flow during diastole are seen.

flow. The flow velocity pattern from collateral vessels is characterized by a slow rise of the systolic phase, widening of the crest time, and absence of distinct second and third deflections. This was similar to the contour of the plethysmograph tracing (Fig. 2). When flow was detected over a stenotic segment a high-pitched sound was usually heard.

In all the adults without evidence of arterial disease normal audible signals and velocity patterns were obtained over all leg arteries. In patients with occlusive peripheral arterial disease abnormal flow velocity patterns were obtained and the site of the altered patterns reflected the location of the occlusion.

The results obtained for the presence of peripheral pulses as demonstrated by palpation and by the ultrasonic detecting device are compared in the Table. In all patients in whom femoral or popliteal pulses could not be felt abnormal flow signals were recorded. In 136 legs in which the posterior tibial 
artery pulse could not be palpated only 14 did not show a flow signal. The dorsalis pedis artery pulse could not be felt in 154 legs and 45 of these did not show flow signals. In eight patients in whom no flow signals were detected on repeated examinations over the dorsalis pedis, posterior tibial, and lateral tarsal arteries, and where the femoral arteriograms showed no run-off from the popliteal artery, amputation was performed.

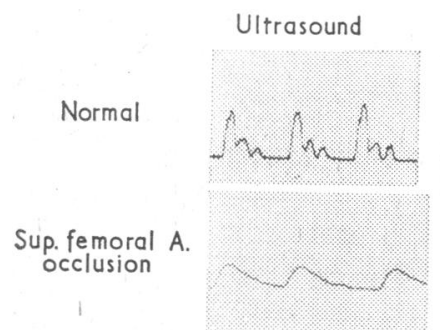

Post. tibial A.

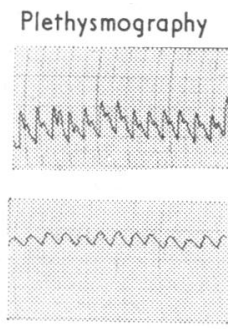

Foot
Fig. 2.-Ultrasonic flow signal and plethysmograph tracing in a normal leg and in a patient with a superficial femoral in a normal leg and in a patient with a superficial femoral artery occlusion. Note the distinct first and second sound of the flow velocity pattern over a normal posterior tibia artery as compared with the dicrotic notch recorded by strain-gauge plethysmography. When there is a superficial femoral artery occlusion the second and third deflections are absent in the ultrasound tracing and the dicrotic notch is absent in the plethysmograph tracing.

Comparison of Leg Pulses as Detected by Palpation and by an Ultrasonic Method in Patients with Occlusive Peripheral Arterial Disease

\begin{tabular}{|c|c|c|c|c|}
\hline \multirow{2}{*}{\multicolumn{2}{|c|}{ Site }} & \multirow{2}{*}{$\begin{array}{c}\text { No. of } \\
\text { Puises } \\
\text { Examined }\end{array}$} & \multicolumn{2}{|c|}{ No. of Pulses Present } \\
\hline & & & On Palpation & $\begin{array}{l}\text { As Detected } \\
\text { by Doppler } \\
\text { Flow Signals }\end{array}$ \\
\hline $\begin{array}{l}\text { Femoral artery } \\
\text { Popliteal artery ... } \\
\text { Posterior tibial artery } \\
\text { Dorsalis pedis artery }\end{array}$ & $\begin{array}{l}\ddot{x} \\
\therefore \\
\therefore\end{array}$ & $\begin{array}{l}200 \\
200 \\
200 \\
200\end{array}$ & $\begin{array}{r}183 \\
139 \\
64 \\
46\end{array}$ & $\begin{array}{l}200 \\
200 \\
122 \\
109\end{array}$ \\
\hline
\end{tabular}

Of 26 patients undergoing direct arterial reconstruction nine had no palpable posterior tibial or dorsalis pedis pulses immediately after the operation. By using the transcutaneous flow detector both audible and written signals were obtained over the posterior tibial and dorsalis pedis arteries in these nine patients; despite the absence of a palpable pulse the configuration of the flow velocity pattern had shown a contour similar to a normal artery, indicating that flow had been restored. This was confirmed by an increase of ankle pressure measured over that segment of the artery (Fig. 3). Twentyfour hours after the operation pedal pulses became palpable and the flow velocity pattern continued to improve and was accompanied by a further increase of ankle pressure, confirming the success of surgery.

\begin{tabular}{|c|c|c|c|c|c|c|}
\hline & \multicolumn{2}{|c|}{ Pre-op. } & \multicolumn{2}{|c|}{ Post--op } & \multicolumn{2}{|c|}{24 hrs. Post.-op. } \\
\hline Pulse & $\begin{array}{l}\text { P.T. } \\
\text { absent }\end{array}$ & $\begin{array}{c}\text { D.P. } \\
\text { absent }\end{array}$ & $\begin{array}{c}\text { P.T. } \\
\text { absent }\end{array}$ & $\begin{array}{c}\text { D.P. } \\
\text { absent }\end{array}$ & $\begin{array}{c}\text { P.T. } \\
\text { present }\end{array}$ & $\begin{array}{c}\text { D.P. } \\
\text { present }\end{array}$ \\
\hline $\begin{array}{l}\text { Flow } \\
\text { velocity } \\
\text { pattern }\end{array}$ & nun & & & chon & & hands \\
\hline $\begin{array}{c}\text { Ankle } \\
\text { pressure } \\
\text { mm.Hg }\end{array}$ & 60 & 60 & 100 & 100 & 125 & 125 \\
\hline
\end{tabular}

FIG. 3.-Changes in the ultrasonic flow velocity patterns recorded over the distal arteries after left iliac endarterectomy. It is seen that immediately after successful operation the ultrasound pattern and ankle pressure have been improved though the pulses could not be palpated at that time.

\section{Discussion}

Franklin et al. (1961) first described an ultrasonic flowmeter based on the Doppler effect which he used in animals. Rushmer et al. (1966) and Strandness et al. (1967) applied this technique in man, but there have been no reports of extensive use of this instrument in the evaluation of peripheral arterial disease.

Our findings have shown that useful information can be obtained with the Parks ultrasonic flow detector provided that the instrument is used correctly; the angle of the probe and its alignment over the underlying artery were critical. When using direct recording on a strip chart constant wave forms were obtained if the maximum intensity of audible signals was determined and this level maintained during recording. When used in a similar manner to a stethoscope by an operator familiar with the instrument the flow signals of collateral, stenotic, and normal pulsatile flow were easily identified. Furthermore, peripheral pulses which were present, but which could not be felt because of ankle oedema or obesity, were instantly detected by this method.

In the present series normal flow signals were obtained in all 50 legs of the normal adults. Patients in whom femoral or popliteal pulses could not be felt all gave abnormal but positive flow signals, indicating the presence of adequate collateral pathways in these limbs. Caution must be observed when interpreting these abnormal flow signals because flow detected over a superficial collateral artery might suggest that a major artery at the same site was patent when, in fact it was occluded. The posterior tibial artery pulse would appear to offer the most reliable information for the lack of adequate collateral pathways in the lower leg and foot; when abnormal flow signals were detected it always indicated arterial pathology. In the eight patients with absent posterior tibial flow signals, and where the dorsalis pedis and lateral tarsal arterial flow signals were also absent, amputation was necessary. When the dorsalis pedis artery pulse is absent a search for a lateral tarsal artery pulse must be made ; Reich (1934) reported this variant of the dorsal pedal artery to be present in $8 \%$. The frequent congenital absence of a dorsalis pedis pulse has made the use of this site unreliable in clinical studies. Barnhorst and Barner (1968) reviewed 1,000 fit children who were less than 10 years old and reported congenital absence of one or both pedal pulses in $12 \%$; the posterior tibial pulses were present in all these children.

Forty-five patients in this study showed no flow signals over the dorsal pedal artery, probably owing either to the congenital absence of the pulse or to the underlying arterial disease.

When pedal pulses were not present by palpation immediately after surgery, the detection of a flow signal over the posterior tibial and dorsalis pedis arteries with a change in the configuration of the flow velocity contour appeared to be most helpful; a change of the flow velocity pattern towards a normal profile accompanied by an increase of ankle pressure suggested the success of surgery.

The transcutaneous Doppler flow velocity detector can be used to examine individual arteries selectively and directly. The technique is painless and harmless and can therefore be repeated at frequent time intervals. The instrument is compact, measuring 17 by 14 by $6 \mathrm{~cm}$. and weighing less than 0.5 $\mathrm{kg}$. It is portable and simple to use. Recent experience (Yao et al., 1968) has shown that when used in conjunction with pressure measurements at the ankle, valuable information can be obtained about the distal arterial bed. Furthermore, the pulse registration by this method allows a reduction of the observer error seen when pulses are determined by palpation. A permanent record of all pulses can be kept for follow-up studies of the progress of the disease and its treatment. 
We are grateful to Mr. Terry Needham, senior technician of the Blood Flow Laboratory of the Surgical Unit at St. Mary's Hospital, for his expert technical assistance in this study. We would like to thank Geistlich Ltd. for the provision of a tape recorder and W. R. Warner \& Co. for other technical assistance.

REFERENCES

Barnhorst, D. A., and Barner, H. B. (1968). New Engl. f. Med., 278, 264
Franklin, D. L., Schlegel, W. A., and Rushmer, R. F. (1961). Science, 134, 564.

Reich, R. S. (1934). Ann. Surg., 99, 613.

Rushmer, R. F., Baker, D. W., and Stegall, H. F. (1966). F. appl. Physiol., 21, 554.

Strandness, D. E., Schultz, R. D., Sumner, D. S., and Rushmer, R. F. (1967). Amer. F. Surg., 113, 311.

Yao, S. T., Hobbs, J. T., and Irvine, W. T. (1968). Presented to XVII European Cardiovascular Congress, London, 1-3 July, 1968.

\title{
Transient Synovitis of Hip. A Virological Investigation
}

\author{
N. J. BLOCKEY,* M.CH.ORTH., F.R.C.S. ; B. B. PORTER, F.R.C.S.
}

Erit. med. 7., 1968, 4, 557-558

Cummary : Virological studies in 17 children with $\checkmark$ transient synovitis of the hip did not confirm the suggestion that this is caused by a viral infection. Minor trauma is thought to be a more likely cause.

\section{Introduction}

Transient synovitis of the hip is a condition of the joint found in children in the age group 2-12 years. It is characterized by sudden development of pain in the hip or thigh, restricted range of joint movement due to muscle spasm, and limp or loss of ability to bear weight on the affected limb; the radiographic appearance is normal, and after rest in bed for several days the symptoms and signs disappear. The resolution, without specific treatment, of acute signs and symptoms within a matter of days is an essential feature of the diagnosis, and so Perthes's disease, tuberculosis, acute rheumatism, etc., are ruled out.

The condition has been described regularly since 1912, has been given many names, and has had many theories proposed for its cause and pathology. Papers by Adams (1963) and de Valderrama (1963) review the literature adequately. Transient synovitis is the name chosen by most writers, and is accurate and non-committal.

\section{Possible Viral Connexion}

The common slight rise in temperature and sedimentation rate, the normal radiographs, the sterile joint fluid, and nonspecific synovial histology (reported by Adams and de Valderrama), combined with the spontaneous cure without antibiotics and the rarity of significant permanent joint change, suggested to us that this disorder might be the result of a virus infection.

Joint symptoms have been described in at least two virus infections. In mumps (Caranasos and Felker, 1967) it has usually been a migratory arthralgia, though monarticular symptoms have been noted. The hip has been affected only half as frequently as other large joints, and males have been involved more often than females. Rubella synovitis has shown a similar pattern (Chambers and Bywaters, 1963), but here adult females have been the most common sufferers and again the hip has been infrequently involved. Lambert (1968) described three cases having multiple joint involvement resembling rheumatic fever in illness due to Mycoplasma pneumoniae infection. In all these three infections complete resolution has occurred.

* Orthopaedic Surgeon, Royal Hospital jor Sick Children, Glasgow C.4. † Orthopaedic Registrar, Western Infirmary, Glasgow W.1.

\section{Investigation}

For a period of eight months (August 1967 to April 1968) all children who on admission were thought to have transient synovitis of the hip were investigated in co-operation with the Regional Virus Laboratory, Ruchill, Glasgow, as follows:

(a) A sample of venous blood was withdrawn, and about three weeks later a second blood sample was taken. The paired sera from each patient were tested by complement fixation for antibodies to the following infective agents: mumps $S$, mumps $V$, herpes simplex, adenovirus group, parainfluenza I, psittacosis-L.G.V., $\mathrm{Q}$ fever, and $M$. pneumoniae. Rubella haemagglutination-inhibition tests were also performed on the sera.

(b) A specimen of stool was inoculated into rhesus monkey kidney and human amnion tissue for culture to detect enterovirus or adenovirus, and suckling mice were injected to detect Coxsackie virus infection.

Twenty-four children were investigated, but seven were later excluded from this diagnosis of transient synovitis; in three the physical signs could not be confirmed, one developed rheumatoid arthritis, one had tuberculosis of the hip, and two turned out to have staphylococcal joint infection. These seven children acted as controls in the virological inquiry.

\section{Clinical Findings}

The 17 children (eight girls and nine boys) who satisfied the criteria for transient synovitis (see Introduction) had clinical features similar to those so often previously described. All were between 2 and 12 years old. There was no predilection for left or right hip. There was a possible history of trauma in four children, and five had a history of recent infection. Eleven had an axillary temperature of $99^{\circ} \mathrm{F}$. $\left(37.2^{\circ} \mathrm{C}\right.$.) or over and 13 had a sedimentation rate of more than $12 \mathrm{~mm}$. in the first hour (Winstrobe). In all children the hip radiographs were normal.

With rest in bed, and in some cases light traction, all recovered in periods of 3 to 10 days.

\section{Virological Findings}

Serology.-Of the 17 patients who had transient synovitis of the hip 16 had the full serological investigation described, as did six of the seven controls. The complement-fixation findings are shown in the Table. Evidence of current infection as proved by a rising antibody titre was found in only two patients with transient synovitis-one to adenovirus group antigen and one 